\title{
Treatment of severe leptospirosis with therapeutic plasma exchange in a pediatric patient
}

\author{
Faruk Ekinci ${ }^{1}$, R. Dinçer Yıldızdaş ${ }^{1}$, Özden Özgür Horoz ${ }^{1}$, Derya Alabaz², İlknur Tolunay ${ }^{1}$, \\ Ercüment Petmezci ${ }^{1}$ \\ Department of Pediatrics, Division of ${ }^{1}$ Pediatric Intensive Care Unit and ${ }^{2}$ Pediatric Infectious Diseases, Çukurova University \\ Faculty of Medicine, Adana, Turkey. E-mail: farukekinci83@gmail.com \\ Received: 5th August 2017, Accepted: 17th September 2017
}

SUMMARY: Ekinci F, Yıldızdaş RD, Horoz ÖÖ, Alabaz D, Tolunay İ, Petmezci E. Treatment of severe leptospirosis with therapeutic plasma exchange in a pediatric patient. Turk J Pediatr 2018; 60: 566-570.

Leptospirosis is a common zoonotic disease caused by spirochetes of the genus Leptospira. Although it is mostly a tropical disease, some case reports have been published from temperate regions of the world. The disease presents with a wide spectrum; from asymptomatic self limited disease to a fatal illness characterized by multi-organ involvement.

An 8-year-old girl presented with a 5-day history of fever, myalgia, fatigue, vomiting and diarrhea. She developed anuria, hypotension and became unconscious one day after admission and was referred to our pediatric intensive care unit for further evaluation and treatment. Initial physical examination revealed fever, jaundice, diffuse petecchiae on whole body, hepatomegaly and severe hypotension. Laboratory investigations showed elevated liver enzymes and bilirubin levels, elevated creatinine and creatine kinase levels and trombocytopenia. The diagnosis of Leptospirosis was detected by rapid IgM test and confirmed by microscopic agglutination test later. She was treated with mechanical ventilation, wide spectrum antibiotics, positive inotropic agents and penicillin $G$ plus two days of continuous renal replacement therapy and five sessions of therapeutic plasma exchange performed daily. She recovered completely and was transferred to the pediatric ward on the $14^{\text {th }}$ day of hospitalization.

The exact role of therapeutic plasma exchange has not been well documented yet, it seems to have benefical effects on clinical and laboratory findings and survival as we observed in our patient and learned from experiences in adult patients presented as case reports.

Key words: leptospirosis, multipl organ failure, therapeutic plasma exchange.

Leptospirosis is a bacterial zoonotic disease caused by spirochetes of the species Leptospira interrogans. The disease is one of the most common, widespread and underdiagnosed infections transmitted from animals to human with a higher incidence in countries with humid subtropical or tropical climates. The natural hosts for this organism are mammals (such as dogs, cats, farm animals, mice and wild rodents) and they excrete infected urine that can remain infectious for years. ${ }^{1}$ Direct contact with urine of infected animals or contaminated water and soil through the broken and watersoaked skin, intact mucous membranes or conjunctiva causes human leptospiral infection. ${ }^{2}$ The disease presents with a wide spectrum: from asymptomatic or subclinical self limited influenza-like disease in which only a positive serological response is detected- to septic shock and multiorgan dysfunction (known as Weil's Disease) which can present with fever, acute kidney injury, pulmonary hemorrhage, acute respiratory distress sydrome (ARDS), bleeding diathesis, impaired hepatic functions, jaundice, trombocytopenia, circulatory collapse and perhaps death. ${ }^{3}$

In leptospirosis, appropriate antibiotic therapy is still the cornerstone of treatment and 
penicillins may shorten the duration of disease if given in the infective phase but supportive measures are also important especially in fulminant disease characterized with septic shock and multi-organ failure ${ }^{4}$. In this paper, we report severe leptospirosis in a pediatric case successfully treated with continuous renal replacement therapy (CRRT) and therapeutic plasma exchange (TPE).

\section{Case Report}

An 8-year-old girl was admitted to a public hospital with a 5-day history of fever $\left(39^{\circ} \mathrm{C}\right)$, myalgia, fatigue, vomiting and diarrhea. She was hospitalized there with a preliminary diagnosis of acute gastroenteritis. She was treated with antipyretics (paracetamol), empiric ceftriaxone and intravenous fluids. During follow-up, she became unconscious, anuric and hypotensive 2 days after admission. On the second day of hospitalization she was referred to our pediatric intensive care unit (PICU) for preliminary diagnosis of acute gastroenteritis and septic shock.

On physical examination at admission, she was unconscious (Glasgow coma scale 6) and intubated with an endotracheal tube. Her temperature was $38.7^{\circ} \mathrm{C}$ (rectal). Initial blood pressure (BP) was 70/35 mmHg (mean:51 $\mathrm{mmHg}$ ) and she was tachycardic (heart rate: 132 beats per minute). Capillary refill time was prolonged at about 4-5 seconds. Her oxygen saturation was $98 \%\left(\mathrm{FiO}_{2}: 60 \%\right)$ and there were wide crackles on her pulmonary examination. There were no lymphadenopathy. The examination of the abdomen was normal except hepatomegaly about $4-5 \mathrm{~cm}$. There were diffuse petechiae on her body, she was icteric and she had marked pretibial edema. The results of initial laboratory testing were as follows: Arterial gases were between normal ranks ( $\mathrm{pH}: 7.37, \mathrm{PO}_{2:} 106 \mathrm{mmHg}, \mathrm{PCO}_{2:} 35$ $\mathrm{mmHg}, \mathrm{HCO}_{3:} 21 \mathrm{mmol} / \mathrm{L}$, base excess: -4 $\mathrm{mmol} / \mathrm{L}$ and lactate was $2 \mathrm{mmol} / \mathrm{L})$. The complete blood count revealed trombocytopenia, leukocytosis (neutrophils, 84\%; lymphocytes, $12 \%$; monocytes, $4 \%$ ) and a hemoglobin level of $9.8 \mathrm{~g} / \mathrm{dl}$ (Table I). The coagulation parameters and other laboratory findings were shown in Table 1. Serum electrolytes, albumin, glucose, amylase, ammonia levels and urinary sediment were normal. Chest radiograph showed bilateral diffuse pulmonary infiltrates. The abdominal ultrasonography showed hepatomegaly about $5 \mathrm{~cm}$ and other findings were normal. Sepsis work-up including blood, urine, endotracheal aspirate and stool cultures were negative. Anti-hepatitis A (HAV) immunglobulin M, anti-hepatitis $\mathrm{C}(\mathrm{HCV})$, anti-HBc and surface antigen hepatitis $B(\mathrm{HBV})$ were negative. Investigations including screening for EpsteinBarr virus (EBV), cytomegalovirus (CMV), human immundeficiency virus (HIV), CrimeanCongo hemorrhagic fever (CCHF), Parvovirus $\mathrm{B} 19$, rubella, toxoplasma, herpes simplex virus, mycoplasma, chlamydia, rickettsia, salmonella and brucella tests were all negative. Antinuclear antibody, anti-ds DNA were negative and serum complement levels (C3, C4) and immunoglobulin levels were within normal limits. Because of the initial presentation and a history of living in a small town and contact with animals, leptospirosis was suspected to be the final diagnosis. Spirochetes were viewed under dark field microscopy directly and serologies for leptospira IgM rapid test was positive with ELISA on the first day. The microscopic agglutination test (MAT) was performed for confirmation of our diagnosis and showed low antibody positivity (1/50) against Leptospira. Control serological testing (MAT) two weeks later was planned.

Vancomycin, ciprofloxacin, amikacin and penicillin G was started immediately. Despite receiving adequate intravenous fluids she was hypotensive, dopamin, dobutamin and noradrenaline infusions were started for circulatory support. Double lumen 9-Fr transient hemodialysis catheter (Duo-flow, Medcomp ${ }^{\circledR}$, Harleysville, PA) was inserted with anatomic landmark approach to right femoral vein. Continuous veno-venous hemodiafiltration (Prismaflex $\AA$, M 60 filter, Gambro Lundia $A B$, Sweden) was started for anuria and fluid overload at the $6^{\text {th }}$ hours after admission. TPE was performed with a centrifugation technique (Spectra Optia; CaridianBCT, Lakewood, CO) on the first day. Total plasma volume (TPV) was calculated manually with this formula: $\mathrm{TPV}=$ total blood volume $\mathrm{X}$ (1-hematocrit). We removed $1.700 \mathrm{ml}$ of dark golden plasma (1.1 times estimated plasma volume) and replaced $1550 \mathrm{ml}$ of fresh frozen plasma on the first day. The plasmapheresis procedure was continued daily for 4 following days, with 5 equal sessions totally. The average removed plasma volume 
was $1530 \mathrm{ml}$ per session (minimum: 1300 $\mathrm{ml}$, maximum: $1640 \mathrm{ml}$ ). The average time of the sessions was 132 min (minimum: 112 min, maximum $153 \mathrm{~min}$ ). We used citrate to prevent clot formation in the extracorporeal circuit and gave $1 \mathrm{ml} / \mathrm{kg}$ calcium gluconate infusion intravenously during each session to prevent hypocalcemia. We used fresh frozen plasma as a replacement fluid in the first session because of abnormal coagulation parameters in her laboratory studies. On the other four procedures we used fresh frozen plasma and $5 \%$ human albumin in $1 / 1$ ratio. No lifethreatening complications were observed during procedures. CRRT was continued for 2 days and was stopped because of the normalization of creatinine levels and spontaneous diuresis on the second day. There was no elevation of creatinine levels or any electrolyte imbalance after stopping CRRT.

After the first session of TPE, the dose of the inotropes were tapered and all inotropes were totally withdrawed after the second session. Trombocytopenia, direct hyperbiluribinemia, pulmonary infiltrates on chest X-ray and mental state improved (Figure 1 and 2) and she was extubated on the $8^{\text {th }}$ day of hospitalization. The antibody titer on the $10^{\text {th }}$ day of hospitalization was 1/200 in MAT (4-times elevation despite
5 sessions of TPE) confirming our diagnosis. She was transferred to the pediatric ward with no sequela on the $14^{\text {th }}$ day of hospitalization to the PICU.

Written informed consent was obtained from the parents for publication of this case report.

\section{Discussion}

Leptospirosis is a common zoonotic disease caused by spirochetes of the genus Leptospira and it is a great health hazard especially in tropical regions. Incidence of the disease in the tropics is approximately 10 times higher than the other parts of the world. ${ }^{5}$ It is not a common disease in our country and is reported sporadically as case reports or series in certain regions of Turkey. ${ }^{6}$

After an incubation period of 2 to 26 days (average 10 days), the disease presents with fever, general malaise, myalgia and headache in $75 \%$ to $100 \%$ of the patients. This initial phase is called infective or septicemic phase and lasts for 4 to 7 days. The clinical course of the disease after these initial symptoms is variable. Most of the cases are mild, subclinical and self limited while some patients $(5-10 \%)$ develop jaundice, pulmonary hemorrhage, ARDS, renal failure, myocarditis, uveitis and rhabdomyolysis

Table I. Laboratory Parameters at Admission and During Hospitalization.

\begin{tabular}{lcccccc}
\hline & $1^{\text {st }}$ day & $2^{\text {nd }}$ day & $3^{\text {rd }}$ day & $5^{\text {th }}$ day & $7^{\text {th }}$ day & $10^{\text {th }}$ day \\
\hline Hemoglobin $(\mathrm{g} / \mathrm{dl})$ & 9.8 & 8.7 & 9.1 & 7.7 & 9.9 & 11.2 \\
White blood cell $\left(/ \mathrm{mm}^{3}\right)$ & 28.000 & 20.090 & 15.560 & 7.400 & 13.170 & 11.200 \\
Thrombocytes $\left(/ \mathrm{mm}^{3}\right)$ & 42.000 & 65.000 & 65.000 & 79.000 & 165.000 & 523.000 \\
Prothrombin time (sec.) & 14.3 & 11.6 & 11 & 12 & 11.3 & 11.7 \\
INR & 1.2 & 0.8 & 0.96 & 1.06 & 1.03 & 1.03 \\
APTT (sec.) & 56 & 32 & 26 & 25 & 21 & 21 \\
Fibrinogen (mg/dl) & 135 & 210 & 128 & 154 & 192 & 232 \\
AST (IU/L) & 248 & 163 & 105 & 72 & 63 & 31 \\
ALT (IU/L) & 165 & 101 & 78 & 62 & 64 & 34 \\
Total bilirubin (mg/dl) & 4.5 & 4.6 & 3 & 1 & - & 0.9 \\
Direct bilirubin(mg/dl) & 3 & 3.2 & 1.6 & 0.3 & - & 0.2 \\
BUN (mg/dl) & 44 & 29 & 22 & 29 & 16 & 16 \\
Creatinine (mg/dl) & 1.15 & 0.75 & 0.45 & 0.44 & 0.33 & 0.3 \\
CK (U/L) & 6309 & 4242 & 1687 & 807 & 142 & 68 \\
Procalcitonin (ng/ml) & 82 & 40 & 10 & 0.5 & - & 0.1 \\
\hline
\end{tabular}

INR: international normalized ratio, APTT: activated partial thromboplastin time, AST: aspartate aminotransferase, ALT: alanine aminotransferase, BUN: blood urea nitrogen, CK: creatine kinase 


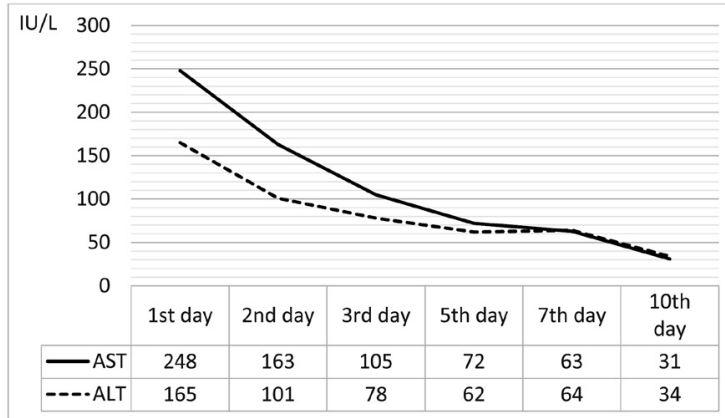

Fig. 1. Serial levels of AST and ALT during hospitalization.

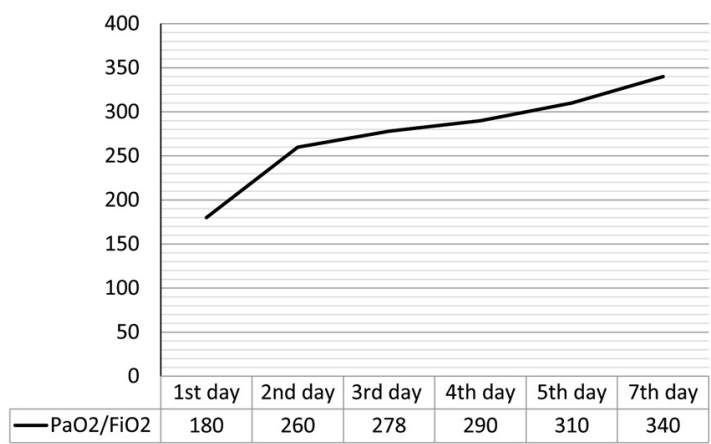

Fig. 2. $\mathrm{PaO}_{2} / \mathrm{FiO}_{2}$ changes during treatment.

known as 'Weil's Disease'. This second phase is the immune phase in which serological tests for anti-Leptospira IgM becomes positive in blood tests. The fatality of the disease ranges from $4 \%$ to $52 \%$ according to the affected organs and systems. ${ }^{7,8}$ Our patient developed septic shock and multi-organ failure 7 days after the initial symptoms of Leptospira infection and this is coinciding with the immune phase of the disease.

Treatment of severe leptospirosis consists of two major components and first step is the choice of appropriate antibiotics. Ceftriaxone or penicillin $\mathrm{G}$ are the main treatment options for severe disease whereas ampicillin, amoxicillin or doxycycline are recommended for mild disease. ${ }^{9}$ Penicillins may shorten the duration and severity of the disesase if given in the early phase of the disease. ${ }^{4}$ In our patient, we gave wide spectrum antibiotics initially, and added penicillin $G$ to the therapy after the positive rapid Leptospira serology test on the first day of hospitalization. The other component is the supportive measures and are as important as appropriate antibiotic therapy. Severe cases with multi-organ involvement should be followed-up in an intensive care unit. Cases with severe pulmonary hemorrhage or ARDS should be mechanically ventilated. Positive inotropic agents should be added to the therapy in patients with circulatory collapse that is unresponsive to intravenous fluids.

CRRT would be an ideal option for patients who developed acute renal failure during the disease. The incidence of acute renal failure is between $10 \%$ to $60 \%$ depending upon the severity of the disease. ${ }^{10}$ The reason of acute renal failure in leptospirosis is tubulointerstitial nephritis associated with direct invasion of the microorganism into kidneys. Other contributing factors are hyperbilirubinemia, endotoxins, hypotension, hypovolemia and rhabdomyolysis. It usually manifests as non-oliguric acute kidney injury ${ }^{11}$. Atypically we observed an oliguric renal failure despite adequate intravenous fluids in our patient and started to perform CRRT in the first day of treatment, lasting for two days. Her urine output improved after renal replacement therapy.

Although leptospirosis is still not included in the seventh edition of the American Society for Apheresisis (ASFA) guidelines for TPE which was published in 2016, therapeutic plasma exchange has been reported as an adjunctive therapy for patients with severe leptospirosis in recent years; especially in adults. ${ }^{12}$ The exact mechanisms of how it works in leptospirosis are not clear yet but we know that it removes circulating endotoxins, bilirubin and inflammatory mediators and prevents immune complex mediated tissue injury which would contribute to a decrease in toxic insult to hepatic, renal, pulmonary and cardiac cells. Taylor et al. ${ }^{12}$ reported an adult patient of 67 years old with severe leptospirosis successfully treated with two sessions of plasma exchange and they highlighted a dramatic reduction of serum bilirubin levels and improvement in mental state and respiratory functions after the second session. Tse et al. ${ }^{13}$ described a dramatic improvement in an adult patient with a single plasma exchange only. Trivedi et al. ${ }^{14}$ reported that plasma exchange with immunosuppression improved survival in patients of pulmonary alveolar haemorrhage due to leptospirosis in a study of 114 adult patients. TPE and CRRT improved systemic and renal hemodynamics in severe cases with renal failure and multiorgan involvement in another study. ${ }^{15}$ Yeşilbaş et al. ${ }^{16}$ reported a 15 -year-old pediatric patient 
with fulminant leptospirosis complicated with macrophage activation syndrome and sclerosing cholangitis successfully treated with CRRT and TPE.

As we searched current literature, our patient is the second pediatric patient in the literature that TPE was performed in severe leptospirosis.

In conclusion, our patient highlights the fact that leptospirosis should be considered as a differential diagnosis in a children with septic shock and multi-organ failure, especially if there is positive history of contact with animals or infected water and in whom jaundice, acute renal failure, rhabdomyolysis and pulmonary involvement seen in the early phase of the disease. Although we cannot determine the exact effect of TPE on our patient's survival, we believe that plasma exchange should be considered as an adjunctive therapy in pediatric cases with fulminant leptospirosis.

\section{REFERENCES}

1. Levett PN. Leptospirosis. Clin Microbiol Rev 2001; 14: 296-326.

2. Chen YS, Cheng SL, Wang HC, Yang PC. Successful treatment of pulmonary hemorrhage associated with leptospirosis and scrub typhus coinfection by early plasma exchange. J Formos Med Assoc 2007; 106 (2 Suppl): S1-S6.

3. American Academy of Pediatrics: Leptospirosis. In: Pickering LK, Baker CJ, Kimberlin DW (ed). Red Book:2012 Report of the Committee on Infectious Diseases. Elk Grove Village, IL: American Academy of Pediatrics; 2012: 469-471.

4. Watt G, Padre LP, Tuazon ML, et al. Placebo-controlled trial of intravenous penicillin for severe and late leptospirosis. Lancet 1988; 1: 433-435.
5. Hartskeerl RA, Collares-Pereira M, Ellis WA. Emergence, control and re-emerging leptospirosis: Dynamics of infection in the changing world. Clin Microbiol Infect 2011; 17: 494-501.

6. Saltoğlu N, Aksu HZ, Tasova Y, et al. Leptospirosis: twelve Turkish patients with the Weil syndrome. Acta Med Okayama 1997; 51: 339-342.

7. Dupont H, Dupont-Perdrizet D, Perie JL, Zehner-Hansen S, Jarrige B, Daijardin JB. Leptospirosis: Prognostic factors associated with mortality. Clin Infect Dis 1997; 25: $720-724$.

8. Pappachan MJ, Mathew S, Aravindan KP, et al. Risk factors for mortality in patients with leptospirosis during an epidemic in Northern Kerala. Natl Med J India 2004; 17: 240-242.

9. Bharti AR, Nally JE, Ricaldi JN, et al; Peru-United States Leptospirosis Consortium. Leptospirosis: A zoonotic disease of global importance. Lancet Infect Dis 2003; 3: 757-771.

10. Singh SS, Vijajachari P, Sinha A. Sugunan AP, Rasheed MA, Sehgal SC. Clinico-epidemiological study of hospitalized cases of severe leptospirosis. Indian J Med Res 1999; 109: 94-99.

11. Yang CW, Wu MS, Pan MJ. Leptospirosis renal disease. Nephrol Dial Transplant 2001; 16(Suppl 5): 73-77.

12. Taylor D, Karamadoukis L. Plasma exchange in severe leptospirosis with multi-organ failure: A case report. J Med Case Rep 2013; 7: 169.

13. Tse KC, Yip PS, Hui KM, et al. Potential benefit of plasma exchange in treatment of severe icteric leptospirosis complicated by acute renal failure. Clin Diagn Lab Immunol. 2002; 9: 482-484.

14. Trivedi SV, Vasava AH, Bhatia LC, Patel TC, Patel NK, Patel NT. Plasma exchange with immunosuppression in pulmonary alveolar haemorrhage due to leptospirosis. Indian J Med Res 2010; 131: 429-433.

15. Siriwanij T, Suttinont C, Tantawichien T, Chusil $S$, Kanjanabuch T, Sitprija V. Haemodynamics in leptospirosis: Effects of plasmapheresis and continuous venovenous haemofiltration. Nephrology (Carlton) 2005; 10: 1-6.

16. Yeşilbaş O, Kıhtır HS, Yıldırım HM, Hatipoğlu N, Sevketoğlu E. Pediatric fulminant leptospirosis complicated by pericardial tamponade, macrophage activation syndrome and sclerosing cholangitis. Balkan Med J 2016; 33: 578-580. 